\title{
EDITORIAL
}

\section{Sepsis: who will shoot first? Pharma or diagnostics?}

\author{
Julien Textoris ${ }^{1,2^{*}}$ (D) and Anthony C. Gordon 3,4
}

(C) 2018 Springer-Verlag GmbH Germany, part of Springer Nature and ESICM

Sepsis has finally been acknowledged as a worldwide issue through a World Health Organization resolution in May 2017 [1, 2]. This was a year ago, but so far very few things have changed. There are $\sim 27$ million patients diagnosed with sepsis each year, and so we-the patients, their relatives and attending physicians-are in desperate need for good news. Although sepsis awareness has increased over the past 5 years, sepsis management still faces several urgent unmet medical needs.

Diagnosis was somewhat put aside with the new sepsis definition [3]: the old SIRS versus SEPSIS dilemma is not so valid anymore and as sepsis is now an infection + organ failure, we should concentrate on (1) pathogen identification and antimicrobial susceptibility testing, plus (2) organ failure diagnosis. Despite the fact that the SOFA score addresses many organs, the immune system is not included, although it accounts for an essential part of the dysregulated host response to infection. Assessing a patient's immune status can be tricky without relevant biomarkers. Hyper-inflammation is associated with poorly specific signs, such as fever and tachycardia, while no clinical signs are associated with immune paralysis.

A better knowledge of the immune state of each patient may dramatically improve the management of septic patients. Several management strategies can be predicated on a precise stratification of a patient's immune status, such as obtaining diagnostic procedures for specific opportunistic pathogens (viruses, fungi), bundles of care (e.g., to reduce ventilator-associated pneumonia), or specific therapies to reverse the immune dysfunction. Patients with hyper-inflammation may benefit from antiinflammatory drugs to prevent the occurrence of additional organ failures, while those with immune paralysis might avoid secondary infections and a prolonged ICU/ hospital stay through the use of immune stimulatory drugs.

Immune modulation is not confidential anymore. Indeed, while retrieving all phase 1-4 interventional sepsis trials currently registered in clinicaltrials.gov, $25 \%$ include manipulations of the host immune response. However, only a few of these are stratifying patients according to a diagnostic test or biomarker result, despite most experts in the field acknowledging that this is the optimal path to follow. The last published trial in the field which assessed the efficacy of interleukin-7 in septic shock patients [4] stratified patients on lymphopenia. There is a rationale for this, as lymphocyte count was also the primary endpoint of the study. However, despite the fact that lymphopenia has been associated with deleterious outcomes $[5,6]$, and that lymphocyte counts are available in most large hospitals, this biomarker has a poor performance in identifying patients with a poor 
prognosis. Indeed, association and prediction are two key features of a pertinent biomarker, but a strong association with one or more conditions does not necessarily mean a good positive/negative predictive value for a later medical event. Let us also consider the expression of HLA-DR on monocytes, which is today the most validated immune paralysis biomarker [7, 8]. It has been associated with poor outcomes (mortality and secondary infections) in several models of injury-induced immunosuppression (sepsis, trauma, burns, etc.) [9, 10]. Yet, despite this valuable performance, its availability is limited mainly to university-associated or large hospitals with expert immunology laboratories. There are multiple reasons for the lack of this test's general availability, but the major one is related to the difficulty in ensuring reproducible results and a standardized protocol using flow cytometry, although Monneret et al. showed a few years ago that such a reproducibility is achievable [11].

In a recent article in Intensive Care Medicine, Conway-Morris et al. reported a large validation study of three, flow-cytometry-based biomarkers (the level of HLA-DR on monocytes, CD88 on neutrophils, and the count of regulatory $\mathrm{T}$-cells), in a multicenter design [12]. They demonstrated that these three cell surface markers were able to risk-stratify patients according to the risk of developing secondary infections. They also have to be congratulated for implementing such a high degree of standardization over several centers and time (approximately 18 months). Indeed, despite the level of association and performances of the given biomarkers, features such as standardization, accuracy, reproducibility, availability, time-to-result, etc. are critical parameters if we want to be able to use these assays in large multicenter clinical trials in sepsis. Conway-Morris A. et al. collaborated closely with BD bioscience, incellDx and AppliedCytometry. The research laboratory of one of this editorial's authors has been a joint research unit between a university hospital (Hospices Civils de Lyon) and an in vitro diagnostic company (bioMérieux) for more than 15 years. Such close collaborations are essential to bring new tools to the market, which respond to unmet medical needs. But a third partner must also be involved: the bio-pharmaceutical companies. In fact, we currently face a vicious circle where pharma companies show a reluctance to set up new clinical trials in sepsis, in part due to the lack of standardized diagnostic tests available to stratify septic patients, while diagnostic companies grumble about investing in the development of such tests if no therapy is available for septic patients. The only way out of this is through large collaborative consortia between academics and bio-pharmaceutical and in vitro diagnostic companies, for instance, as in the ongoing REALISM study (NCT02638779) [13].
Many funding bodies encourage such collaboration to ensure a true and efficient translation between research and clinical practice. The study by Conway-Morris and colleagues was in part funded by a UK government grant designed to facilitate this type of collaboration. This naturally led the discussion towards conflicts of interests between health care professionals and industry. The latter has been deeply involved in providing a clear code of conduct to tackle such issues. Recently, MedTech Europe's Code of Ethical Business Practice led to stronger European regulations. Clear rules ensure ethical relationships regarding grants, consultants, and research collaborations (among other things). Transparency is probably the most important criterion, but government bodies and institutions should ensure that the burden of administrative work required to achieve such transparency does not prevent such relationships. All these detailed rules may sometimes seem confusing. Creating and maintaining such an ethical culture requires training both in the private and public sectors. Promoting a debate within the institutions or communities of health-care providers on how to make these collaborations work would be extremely valuable.

The study by Conway-Morris et al. shows that the implementation of rigorous protocols and standardization of sepsis biomarkers are achievable. Targeting immune paralysis in a personalized trial therefore seems feasible. However, such flow-cytometry-based approaches still currently limit inclusions to large/ university centers where such expertise is available. More accessible techniques will be needed to allow the implementation of immunotherapy more widely (e.g., CD74 mRNA expression by quantitative PCR, which has recently been validated as a molecular surrogate to mHLA-DR [14]). Moreover, given the complexity of the immune system, setting up a sepsis trial which truly addresses immune dysfunction in a personalized manner (such as the ongoing PROVIDE study, NCT03332225) will require assessment of both the innate and adaptive immune systems, and the extent of both the pro- and anti-inflammatory responses. This will likely require the quantification of multiple biomarkers-perhaps 5,10 , or more-at the same time in a single sample [15]. Molecular biology is currently an interesting option as it allows such a multiplexing capacity, along with high standardization and automation, and is increasingly widely available. Finally, the ability to assess the immune status of critically ill patients will facilitate the adoption of adaptive clinical trials, allowing enrichment and stratification of patients who will be helped most by specific management approaches and treatments, as is now being carried out in other medical specialties [16]. 


\section{Author details}

${ }^{1}$ Joint Research Unit, Hospices Civils de Lyon-bioMérieux-Université Claude Bernard Lyon 1, EA7426"Pathophysiology of Injury-Induced Immunosuppression (PI3)", Hôpital E. Herriot, Lyon, France. ${ }^{2}$ Anesthesiology and Critical Care Medicine, Hospices Civils de Lyon-Université Claude Bernard Lyon 1, Lyon, France. ${ }^{3}$ Section of Anaesthetics, Pain Medicine and Intensive Care, Department of Surgery and Cancer, Faculty of Medicine, Imperial College London, London, UK. ${ }^{4}$ Intensive Care Unit, St Mary's Hospital, Imperial College Healthcare NHS Trust, London, UK.

\section{Compliance with ethical standards}

\section{Conflicts of interest}

Julien Textoris is a part-time employee of an in vitro diagnostic company, bioMérieux, and part-time employee of Hospices Civils de Lyon. Both institutions hold patents related to sepsis biomarkers and JT is inventor on some of them. The views presented in this editorial are JT's personal opinion and do not necessarily represent the viewpoint, strategy or opinions of bioMérieux. Anthony Gordon is supported by an NIHR Research Professorship (RP-2015-06-018) and the NIHR Imperial Biomedical Research Centre. The views expressed are those of the authors and not necessarily those of the NHS, the NIHR or the department of health. He also reports that he has received speaker fees from Orion Corporation Orion Pharma and Amomed Pharma. He has consulted for Ferring Pharmaceuticals, Tenax Therapeutics, Baxter Healthcare, Bristol-Myers Squibb and GSK, and received grant support from Orion Corporation Orion Pharma, Tenax Therapeutics and HCA International with funds paid to his institution.

\section{Received: 15 May 2018 Accepted: 16 May 2018}

Published online: 14 June 2018

\section{References}

1. Seventieth World Health Assembly (2017) Improving the prevention, diagnosis and clinical management of sepsis. WHA70.7

2. Reinhart K, Daniels R, Kissoon N, Machado FR, Schachter RD, Finfer S (2017) Recognizing sepsis as a global health priority - a WHO resolution. N Engl J Med 377:414-417

3. Singer M (2016) The new sepsis consensus definitions (sepsis-3): the good, the not-so-bad, and the actually-quite-pretty. Intensive Care Med 42:2027-2029

4. Francois $B$, Jeannet R, Daix T, Walton AH, Shotwell MS, Unsinger J, Hotchkiss RS (2018) Interleukin-7 restores lymphocytes in septic shock: the IRIS-7 randomized clinical trial. JCI Insight 3:e98960. https://doi. org/10.1172/jci.insight.98960
5. Daix T, Guerin E, Tavernier E, Mercier E, Gissot V, Herault O, Francois B, the Septiflux trial group (2018) Multicentric standardized flow cytometry routine assessment of septic patient to predict clinical worsening. Chest. https://doi.org/10.1016/j.chest.2018.03.058

6. Drewry AM, Samra N, Skrupky LP, Fuller BM, Compton SM, Hotchkiss RS (2014) Persistent lymphopenia after diagnosis of sepsis predicts mortality. Shock 42:383-391

7. Landelle C, Lepape A, Voirin N, Tognet E, Venet F, Bohe J, Monneret G (2010) Low monocyte human leukocyte antigen-DR is independently associated with nosocomial infections after septic shock. Intensive Care Med 36:1859-1866

8. Venet F, Rimmele T, Monneret G (2018) Management of sepsis-induced immunosuppression. Crit Care Clin 34:97-106

9. Gouel-Cheron A, Allaouchiche B, Floccard B, Rimmele T, Monneret G (2015) Early daily mHLA-DR monitoring predicts forthcoming sepsis in severe trauma patients. Intensive Care Med 41:2229-2230

10. Venet F, Tissot S, Debard AL, Faudot C, Crampe C, Pachot A, Monneret G (2007) Decreased monocyte human leukocyte antigen-DR expression after severe burn injury: correlation with severity and secondary septic shock. Crit Care Med 35:1910-1917

11. Demaret J, Walencik A, Jacob MC, Timsit JF, Venet F, Lepape A, Monneret G (2013) Inter-laboratory assessment of flow cytometric monocyte HLADR expression in clinical samples. Cytometry B 84:59-62

12. Morris AC, Datta D, Shankar-Hari M, Stephen J, Weir CJ, Rennie J, Antonelli J, Bateman A, Warner N, Judge K, Keenan J, Wang A, Burpee T, Brown KA, Lewis SM, Mare T, Roy Al, Hulme G, Dimmick I, Rossi AG, Simpson AJ, Walsh TS (2018) Cell-surface signatures of immune dysfunction riskstratify critically ill patients: INFECT study. Intensive Care Med. https://doi. org/10.1007/s00134-018-5247-0

13. Rol ML, Venet F, Rimmele T, Moucadel V, Cortez P, Quemeneur L, Monneret G, the REALISM study group (2017) The REAnimation low immune status markers (REALISM) project: a protocol for broad characterisation and follow-up of injury-induced immunosuppression in intensive care unit (ICU) critically ill patients. BMJ Open 7:e015734

14. Peronnet E, Venet F, Maucort-Boulch D, Friggeri A, Cour M, Argaud L, Lepape A, the MIP Réa study group (2017) Association between mRNA expression of CD74 and IL10 and risk of ICU-acquired infections: a multicenter cohort study. Intensive Care Med 43:1013-1020

15. Davenport EE, Burnham KL, Radhakrishnan J, Humburg P, Hutton P, Mills TC, Knight JC (2016) Genomic landscape of the individual host response and outcomes in sepsis: a prospective cohort study. Lancet Respir Med 4:259-271

16. Park JW, Liu MC, Yee D, Yau C, van't Veer LJ, Symmans WF, Berry DA, I-SPY Investigators (2016) Adaptive randomization of neratinib in early breast cancer. N Engl J Med 375:11-22 